\title{
Special Section on:
}

\section{Recent advances in the Intelligence technology and Security applications}

\author{
Guest Editor: Dengpan Ye
}

Intelligence technology can be applied to searching the Internet and data mining, interpreting Internet-derived material, the human Web interface, remote condition monitoring and many other areas. It has become one of the key issues on our way to a knowledge society. Currently, computer systems in large-scale, decentralized and heterogeneous environments are now facing various threats including viruses and other malware. Security research seeks to make computers safer and less vulnerable to those attacks. Therefore, a security system is expected to be built in innovative applications allowing users to enjoy more scalable and comprehensive services while preserving trust, security and privacy at the same time.

Much research work has been done in different fields such as intelligence computing and trusted computing. Our aim has been to combine these fields for research in information security. This special section aims to expose the latest research results on combined intelligence computing and security applications. It is composed of 7 refereed papers covering such topics as Secure intelligent Sensor Localization, Intelligent Objects Detection, trusted virtual machine, Scalable intelligent Authentication, Access Control, Intelligent Risk Analysis and Multi-Objective Optimization problems. The section is expected to demonstrate new work in the field of computational intelligence in information security and trust, investigate novel solutions and discuss future trends in this field.

The Guest Editor wish to express our deep appreciation to the authors for their contribution, to the reviewers for their careful, insightful and constructive reviews that led to further improvement of the articles. We are thankful to Mr Zeger Karssen, Publishing Director at Atlantis Press and to Prof. Jie Lu and Prof. Luis Lopèz, Editors-in-Chief of the Journal, for their assistance throughout the publication process.

Guest Editor: Associate Prof. Dengpan Ye

School of Computer

Wuhan University

Wuhan 430072, P R China

E-mail: yedp@whu.edu.cn 